\title{
Identification of dysregulated modules based on network entropy in type 1 diabetes
}

\author{
YAN ZHENG $^{1}$, LIWEI LIU $^{2}$ and JIFENG YE ${ }^{3}$ \\ ${ }^{1}$ Department of Anus and Intestine Surgery, Weifang People's Hospital, Weifang, Shandong 261000; ${ }^{2}$ Department of \\ Health Management, The Affiliated Central Hospital of Qingdao University, Qingdao, Shandong 266000; ${ }^{3}$ Department of \\ Endocrinology and Metabolism, The Second People's Hospital of Liaocheng, Liaocheng, Shandong 252601, P.R. China
}

Received July 3, 2017; Accepted October 31, 2017

DOI: $10.3892 /$ etm.2018.5803

\begin{abstract}
Type 1 diabetes is a prevalent autoimmune disease of which the underlying mechanisms remain to be elucidated. The aim of the study was to identify dysregulated modules of type 1 diabetes. After microarray data were preprocessed, 20,545 genes were obtained. By integrating gene expression data and protein-protein interactions (PPI) data, 48,778 new networks were obtained, including 7,953 genes. After simplifying networks, we obtained 24 target networks. By ranking networks with $\mathrm{P}$-values, two modules with $\mathrm{P}<0.05$ were identified, including the genes, CCNB1, CDC45, GINS2, NDC80, FBXO5, NCAPG and DLGAP5. Module 2 was part of module 1 . The identified modules and genes may provide new insights into the underlying biological mechanisms that drive the progression of type 1 diabetes.
\end{abstract}

\section{Introduction}

Type 1 diabetes is an autoimmune disease characterized by the $\mathrm{T}$ cell-mediated destruction of insulin-producing $\beta$-cells in the islets of Langerhans (1). Type 1 diabetes is one of the most common chronic diseases of childhood (2), particularly in boys (3). Despite recent broad organisational, intellectual and fiscal investments, there is no valid method to prevent or cure type 1 diabetes. Therefore, elucidating the mechanisms of type 1 diabetes is critical for the clinical diagnosis and treatment for type 1 diabetes. Thus the aim of the present study was to explore molecular mechanisms of type 1 diabetes.

Determinants of diabetes pathology are complex, including environment factors and genetic factors. It is generally accepted that environmental agents initiated the pathologic process in type 1 diabetes, as many cases are diagnosed in autumn

Correspondence to: Dr Jifeng Ye, Department of Endocrinology and Metabolism, The Second People's Hospital of Liaocheng, 306 Jiankang Road, Linqing, Liaocheng, Shandong 252601, P.R. China E-mail: ffy255@163.com

Key words: dysregulated module, entropy, network, type 1 diabetes, protein-protein interaction and winter (4). Birth during spring is also associated with a higher chance of having type 1 diabetes (5). Efforts have been made on modules to describe the influence of environment on type 1 diabetes, including the gut microbiome (6) and hygiene hypothesis (3). However, no specific agents have been identified with an unequivocal influence on pathogenesis. Type 1 diabetes is clearly a polygenic disorder, with 50 susceptibility regions having been identified (3), of which the human leukocyte antigen (7) region on chromosome 6 potentially provides half of the genetic susceptibility, especially HLA class II alleles (3). Most of the associated loci are thought to be involved in immune responses (8). According to the literature (3), the associated SNPs are localized to enhancer sequences active in thymus, $\mathrm{T}$ and $\mathrm{B}$ cells, and $\mathrm{CD} 34^{+}$stem cells.

Although there has been considerable research on type 1 diabetes progression, the data are huge and complex. A networkbased approach was suggested as a powerful tool for studying the complex behavior of biological systems (3). To elucidate the molecular mechanisms of type 1 diabetes, we introduced a new method to screen differential modules between the disease and normal groups. We downloaded gene expression data of type 1 diabetes from the Array Express database. By combining gene expression data and protein-protein interactions (PPI) data, we constructed target networks. Local entropy and global entropy of network were calculated to screen differential modules between diabetes and normal group.

\section{Materials and methods}

Gene expression data. Microarray data of E-GEOD-10586 (3), along with its annotation files, was downloaded from the Array Express database. The data included 12 diabetes patients and 15 healthy controls. The platform in the present study was A-AFFY-44-Affymetrix GeneChip Human Genome U133 Plus 2.0 [HG-U133_Plus_2].

Data preprocessing. Microarray data were preprocessed as follows. To eliminate the influences of non-specific hybridization, background was corrected with robust multichip average (RMA) (3). After data were normalized with 'quantiles' (9), perfect match (3)/mismatch (MM) correction was conducted using the MAS method (10), and median polish was conducted for summarizing data (11). Microarray data 
were then transformed into an expression set. According to the gene ID and symbol in the annotation file of the platform, the gene ID was changed to its probe ID. Finally, the expression profiles with 20,545 genes were obtained.

PPI networks construction. Human-associated PPI data were downloaded from Search Tool for the Retrieval of Interacting Genes/Proteins (STRING) database (12), including 16,730 genes and 1,048,576 pairs of interactions. Protein ID was converted to a gene symbol. Self-loops and proteins without expression value were removed. The combine-score was used to examine the relationship between two genes. To selected PPIs with closer relationship, we set the criteria of combined score $\geq 0.8$, generating a new PPI network, including 8,590 nodes and 53,975 edges.

Gene interactions in the PPI network were reweighed using the Pearson's correlation coefficient. The absolute value of each interaction was considered as the interactive-score. Finally, PPI networks of the normal and diabetes group were calculated using the interactive-scores.

Comparison and identification of modules. To construct networks in the disease and normal groups, we applied a module-identification algorithm, which is based on cliquemerging according to Srihari and Ragan (13).

The algorithm calculations included two steps: Firstly, it finds all maximal cliques from the PPI networks of the normal and diabetes groups. Maximal cliques $(26,580)$ were found in both groups and ranked in non-increasing order of their weighted interaction densities. Secondly, the cliques were ranked according to their weighted interaction density (3) and merged or removed highly overlapping cliques The score of a clique $\mathrm{C}$ was defined as its weighted interaction density,

$$
\text { score }(\mathrm{C})=\frac{\sum_{i, j \in C} \omega(\mathrm{i}, \mathrm{j})}{|\mathrm{C}| *(|\mathrm{C}|-1)}
$$

where $\omega(i, j)$ indicates the weight of the interaction between $\mathrm{i}$ and $\mathrm{j}$ calculated using fast depth-first method (14).

In total 8,002 maximal cliques were identified in a PPI network, and the overlapped cliques should be removed. The inter-connectivity between two cliques was used to determine whether two overlapped cliques should be merged together. The inter-connectivity between the non-overlapping proteins of $\mathrm{C} 1$ and $\mathrm{C} 2$ was calculated as

$$
\begin{aligned}
& \text { inter-score,C1,C2.=,-,, } u \in(C 1-C 2)--, v \in C 2--w(u, v) . .-, \\
& C 1-C 2 . ;, C 2 . . \cdot, u \in(C 2-C 1)--, v \in C 1--w(u, v) . .-, C 2-C 1 . \cdot, C 1 \ldots
\end{aligned}
$$

Given a set of cliques ranked in descending order of their score, denoted as $\{\mathrm{C} 1, \mathrm{C} 2, \ldots, \mathrm{Ck}\}$, clustering based on the maximal cliques (CMC) algorithm was removed and merged highly overlapped cliques as follows. For every clique $\mathrm{Ci}$, if there existed a clique $\mathrm{Cj}$ such that $\mathrm{Cj}$ had a lower score than $\mathrm{Ci}$ and

$$
\left|\mathrm{C}_{\mathrm{i}} \cap \mathrm{C}_{\mathrm{j}}\right| /\left|\mathrm{C}_{\mathrm{j}}\right| \geq \text { overlap_threshold }\left(\mathrm{t}_{\mathrm{o}}\right) \text {, }
$$

where overlap-threshold was a predefined threshold for overlapping. Subsequently, the weighted interconnecting score of different nodes in the two cliques was calculated. If such $\mathrm{Cj}$ existed, then the interconnectivity score between $\mathrm{Ci}$ and $\mathrm{Cj}$ was used to decide whether to remove $\mathrm{Cj}$ or merge $\mathrm{Cj}$ with $\mathrm{Ci}$. If inter-score $(\mathrm{Ci}, \mathrm{Cj})$ and merge-threshold $\left(\mathrm{t}_{\mathrm{m}}\right)$ existed, then $\mathrm{Cj}$ was merged with $\mathrm{Ci}$ to form a module; otherwise, $\mathrm{Cj}$ was removed. In this study, the overlap-threshold was set to 0.5 and merge-threshold was set to 0.25 .

Identification of differential modules. To identify differential modules between disease group and normal group, we constructed target networks and performed Wilcoxon rank sum test (15).

Comparing modules across condition. To search for similar or the same modules between the normal and diabetes groups, module correlation densities for modules were calculated. Let $\mathrm{S}=\left\{\mathrm{S}_{1}, \mathrm{~S}_{2}, \ldots, \mathrm{S}_{\mathrm{n}}\right\}$ and $\mathrm{T}=\left\{\mathrm{T}_{1}, \mathrm{~T}_{2}, \ldots, \mathrm{T}_{\mathrm{m}}\right\}$ be the sets of modules identified from the normal and disease networks, respectively. For each $\mathrm{Si} \in \mathrm{S}$, module correlation densities were calculated as:

$$
\mathrm{d}_{c \mathrm{c}}\left(\mathrm{S}_{\mathrm{i}}\right)=\frac{\sum_{\mathrm{p}, \mathrm{q} \in \mathrm{S}_{\mathrm{i}}} \operatorname{PCC}((\mathrm{p}, \mathrm{q}), \mathrm{N})}{\left(\frac{\mathrm{S}_{\mathrm{i}} \mid}{2}\right)}
$$

where $(p, q)$ is a protein pair, $\operatorname{PCC}((p, q), N)$ is the Pearson's correlation of $(p, q)$ under normal conditions, and $S_{i}$ is the $i$-th modules identified from networks.

The correlation densities for disease modules were calculated similarly.

After all the modules were examined, 69 pairs of similar or identical modules were identified.

Construction of target network. Shared genes and interactions in the normal and disease modules were reserved, generating a new network, designated as target network. Finally, 24 target networks were identified.

To compare the network features of the target networks, network entropy was conducted in this study (16).

The local network entropy of a node $\mathrm{i}$, denoted $\mathrm{S}_{\mathrm{i}}$, is defined as

$$
S_{i}=-\frac{1}{\log k_{i}} \sum_{j \in N_{i}} p_{i, j} \log p_{i, j}
$$

where $k_{i}$ is the degree of node $i, N(i)$ is the set of neighbor nodes of node $\mathrm{i}$ and $\mathrm{p}_{\mathrm{ij}}$ defines a stochastic probability matrix on the network, which is defined by

$$
p_{i j}=\frac{\left|c_{i j}\right|}{\sum_{k \in N_{i}} c_{i k}}
$$

where $c_{i j}$ is the Pearson's correlation coefficient (PCC) between protein $\mathrm{i}$ and protein $\mathrm{j}$.

The global network entropy, denoted S, was defined as follows:

$$
\mathrm{S}=\sum_{\mathrm{i}=1}^{\mathrm{n}} \mathrm{C}_{\mathrm{i}} \mathrm{S}_{\mathrm{i}}
$$

where $\mathrm{n}$ is the total number of nodes in the network, and $\mathrm{C}_{\mathrm{i}}$ is the degree centrality of node $i$.

$$
C_{i}=\frac{k_{i}}{n-1}
$$


The differential network entropy was defined as follows: $(7)$

$$
\Delta S_{i}=S_{i}^{I}-S_{i}^{N}
$$

where $\mathrm{S}_{\mathrm{i}}^{\mathrm{I}} \mathrm{S}^{\mathrm{N}}{ }_{\mathrm{i}}$ is the local network entropy of node $\mathrm{i}$ in the disease and normal networks, respectively.

Significant test. To determine whether the distributions of local network entropy of the disease and normal networks were significantly different, we performed the non-parametric one-tailed Wilcoxon rank sum test (15).

The disease sample labels were permuted and global entropy of networks in the disease and normal groups were recalculated. This process was repeated $\mathrm{L}$ times. P-value of the test was used as a measure of the degree of difference between the values in the two networks. P-value was calculated as:

$$
\left\{\# \mid S_{1}^{\mathrm{I}} \leq \mathrm{S}_{\mathrm{obs}}^{\mathrm{N}} \text {, for } \mathrm{l}=1, \ldots, \mathrm{L}\right\} / \mathrm{L}
$$

$\mathrm{P}<0.05$ was considered to indicate a statistically significant difference.

\section{Results}

Gene expression data. After data preprocessing, 20,545 genes were obtained. Proteins from PPI networks were transformed to a gene symbol. Based on the criteria of combined score $\geq 0.8,53,975$ interactions and 8,590 nodes were obtained. The interactions between genes and PPI networks were investigated. The interactions existing in the PPI and gene expression data were reserved. In total, 48,778 new PPI interactions, including 7,953 genes were obtained.

Identifying dysregulated module. Modules constituted shared genes in the disease and normal groups were regarded as target networks. In total, 24 networks were obtained. Global entropy of networks was calculated from local entropy of nodes. After significance test, P-values of networks were obtained. Two significant differential modules were identified with $\mathrm{P}<0.05$ (Table I). Module 1 was constructed with 7 genes and 21 interactions (Fig. 1). Each gene interacted with other genes. Module 2 was constructed with 4 genes and 6 interactions (Fig. 2). Module 2 was part of module 1, as the 4 genes, NDC80, FBXO5, NCAPG and DLGAP5, were identified in module 1 .

\section{Discussion}

In this study, by integrating gene expression data and PPI, we identified 48,778 new PPI interactions, including 7,953 genes. In the network analysis, 24 target modules were identified. In the entropy analysis, two differential modules between type 1 diabetes group and normal group were obtained, and module 2 was part of module 1 . Therefore, module 1 which was constructed with 7 genes, including $C C N B 1, C D C 45$, GINS2, NDC80, FBXO5, NCAPG and DLGAP5 was the most significant module. It was suggested to help understanding the mechanism of type 1 diabetes.
Table I. Two modules with $\mathrm{P}<0.05$ were identified.

\begin{tabular}{lcc}
\hline Module & $\Delta S$ & P-value \\
\hline 1 & 0.2429241 & 0.043 \\
2 & 0.1019656 & 0.025 \\
\hline
\end{tabular}

$\Delta \mathrm{S}$ indicates differential network entropy between normal and disease group.

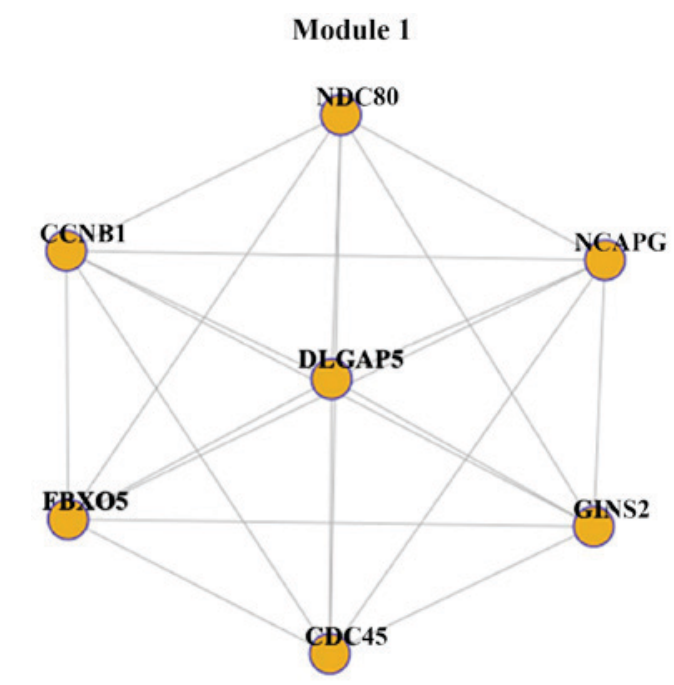

Figure 1. Dysregulated module 1.

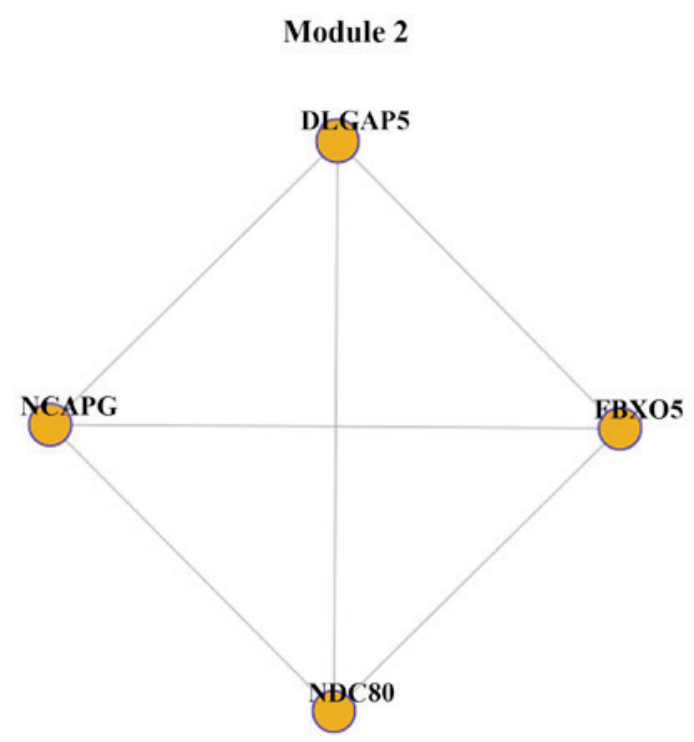

Figure 2. Dysregulated module 2.

This method is based on network entropy, which performs better than other network metrics in characterizing the inflammatory network as proposed by Jin et al (16).

$C C N B 1$ is a gene expressing a regulatory protein, cyclin $\mathrm{B} 1$, which forms a complex with p34 (Cdk1) to form the maturationpromoting factor (MPF). Once activated by dephosphorylation by the phosphatase $\mathrm{Cdc} 25$, the complex promotes several events of early mitosis (18). It has been found that $C C N B 1$ was 
significantly upregulated in non-obese diabetic mesenchymal stem cells and proposed that genetic variants in CCNB1 were associated with increased reporter gene expression through binding of transcription factors nuclear factor-Y, which elevated fasting plasma glucose in humans (3). By contrast, in the non-obese diabetic mouse study, NDC80, CCNB1, FBXO5, $N C A P G$ and $C D C 45$ (19) were involved in cell cycle, which promoted the development of type 1 diabetes mellitus (3).

Although no evidence showed that GINS2 was correlated with diabetes, its expression was downregulated by high glucose in retinal pigment epithelial cell lines (3).

NDC80 encodes a component of the NDC80 kinetochore complex, which functions to organize and stabilize microtubule-kinetochore interactions and is required for proper chromosome segregation (NCBI Gene Database). It was presented that the NUF2 gene, which also encodes a component of the NDC80 kinetochore complex, was upregulated in diabetes HUVEC compared with normal HUVEC (3). Thus $N D C 80$ may play a similar role in diabetes.

$F B X O 5$ encodes a member of the F-box protein family. FBXO5 was detected to duplicate in chromosome observed from 15 patients with Mayer-Rokitansky-Kuster-Hauser syndrome (3). By contrast, diabetes has been reported to cause malformations of Mullerian ducts in females (20). Therefore, we suggested that $F B X O 5$ may also function in diabetes.

$N C A P G$ encodes a component of condensin I, which is a large protein complex involved in chromosome condensation. Several single nucleotide polymorphisms (SNPs) near the gene of $N C A P G$ were associated with type 2 diabetes (3). However, whether the gene plays a key role in type 1 diabetes still needs further study.

$D L G A P 5$ encodes a kinetochore protein that stabilizes microtubules in the vicinity of chromosomes. In adrenocortical tumors, DLGAP5 was identified as a diagnostic marker since it was differentially expressed between recurring and non-recurring adrenocortical tumors (3). However, in diabetes, no studies have shown the functions of DLGAP5.

In conclusion, one dysregulated module was identified using the network-based entropy analysis, which was considered to play a key role in type 1 diabetes progression. It is suggested that this module may function as a therapeutic indicator for type 1 diabetes. Nevertheless, there are limitations to the present study. The sample size was not large enough to affect the conclusions to some degree. Additionally, the results need more clinical evidence for further validation.

\section{Competing interests}

The authors declare that they have no competing interests.

\section{References}

1. Roep BO and Peakman M: Diabetogenic T lymphocytes in human type 1 diabetes. Curr Opin Immunol 23: 746-753, 2011.

2. Gale EA: Type 1 diabetes in the young: The harvest of sorrow goes on. Diabetologia 48: 1435-1438, 2005.
3. Baidal DA, Ricordi C, Garcia-Contreras M, Sonnino A and Fabbri A: Combination high-dose omega-3 fatty acids and high-dose cholecalciferol in new onset type 1 diabetes: A potential role in preservation of beta-cell mass. Eur Rev Med Pharmacol Sci 20: 3313-3318, 2016.

4. Kalliora MI, Vazeou A, Delis D, Bozas E, Thymelli I and Bartsocas CS: Seasonal variation of type 1 diabetes mellitus diagnosis in Greek children. Hormones (Athens) 10: 67-71, 2011.

5. Atkinson MA, Eisenbarth GS and Michels AW: Type 1 diabetes. Lancet 383: 69-82, 2014.

6. Boerner BP and Sarvetnick NE: Type 1 diabetes: Role of intestinal microbiome in humans and mice. Ann NY Acad Sci 1243: 103-118, 2011.

7. Carlsson P and Mahlapuu M: Forkhead transcription factors: Key players in development and metabolism. Dev Biol 250: 1-23, 2002.

8. Concannon P, Rich SS and Nepom GT: Genetics of type 1A diabetes. N Engl J Med 360: 1646-1654, 2009.

9. Rifai $\mathrm{N}$ and Ridker PM: Proposed cardiovascular risk assessment algorithm using high-sensitivity C-reactive protein and lipid screening. Clin Chem 47: 28-30, 2001.

10. Bolstad BM, Irizarry RA, Astrand M and Speed TP: A comparison of normalization methods for high density oligonucleotide array data based on variance and bias. Bioinformatics 19: 185-193, 2003.

11. Irizarry RA, Bolstad BM, Collin F, Cope LM, Hobbs B and Speed TP: Summaries of Affymetrix GeneChip probe level data. Nucleic Acids Res 31: e15, 2003.

12. Szklarczyk D, Franceschini A, Kuhn M, Simonovic M, Roth A, Minguez P, Doerks T, Stark M, Muller J, Bork P, et al: The STRING database in 2011: Functional interaction networks of proteins, globally integrated and scored. Nucleic Acids Res 39: D561-D568, 2011.

13. Srihari S and Ragan MA: Systematic tracking of dysregulated modules identifies novel genes in cancer. Bioinformatics 29: 1553-1561, 2013.

14. Tomita E, Tanaka A and Takahashi H: The worst-case time complexity for generating all maximal cliques and computational experiments. Theor Comput Sci 363: 28-42, 2006.

15. Rosner B, Glynn RJ and Lee ML: Incorporation of clustering effects for the Wilcoxon rank sum test: A large-sample approach. Biometrics 59: 1089-1098, 2003.

16. Jin S, Li Y, Pan R and Zou X: Characterizing and controlling the inflammatory network during influenza A virus infection. Sci Rep 4: 3799, 2014.

17. Ma H, Hostuttler M, Wei H, Rexroad CE III and Yao J: Characterization of the rainbow trout egg microRNA transcriptome. PLoS One 7: e39649, 2012.

18. Berry LD and Gould KL: Regulation of Cdc2 activity by phosphorylation at T14/Y15. Prog Cell Cycle Res 2: 99-105, 1996.

19. Pollok S, Bauerschmidt C, Sänger J, Nasheuer HP and Grosse F: Human Cdc45 is a proliferation-associated antigen. FEBS J 274: 3669-3684, 2007.

20. Iwasaki N, Okabe I, Momoi MY, Ohashi H, Ogata M and Iwamoto Y: Splice site mutation in the hepatocyte nuclear factor-1 beta gene, IVS2nt $+1 \mathrm{G}>\mathrm{A}$, associated with maturityonset diabetes of the young, renal dysplasia and bicornuate uterus. Diabetologia 44: 387-388, 2001.

(i) (3) This work is licensed under a Creative Commons Attribution-NonCommercial-NoDerivatives 4.0 International (CC BY-NC-ND 4.0) License. 